\title{
Doppler angle correction in the measurement of intrarenal parameters
}

This article was published in the following Dove Press journal: International Journal of Nephrology and Renovascular Disease 26 March 2011

Number of times this article has been viewed

\author{
Jing Gao' \\ Keith Hentel' \\ Qiang Zhu ${ }^{2}$ \\ Teng $\mathrm{Ma}^{2}$ \\ George Shih' \\ Kevin Mennitt' \\ Robert Min' \\ 'Department of Radiology, New York \\ Presbyterian Hospital, Weill Cornell \\ Medical College, NY, USA; ${ }^{2}$ Division \\ of Diagnostic Ultrasound, Department \\ of Radiology, Beijing Tongren Hospital, \\ Capital Medical University, \\ Beijing, China
}

Correspondence: Jing Gao

Department of Radiology, New York Presbyterian Hospital, Weill Cornell Medical Center, 525 East 68th Street, St 8A27, New York, NY 10065, USA

$\mathrm{Tel}+\mathrm{I} 2127462342$

Fax +I 2127468596

Email jig200।@med.cornell.edu
Background: The aim of this study was to assess differences in intrarenal artery Doppler parameters measured without and with Doppler angle correction.

Methods: We retrospectively reviewed color duplex sonography in 30 normally functioning kidneys (20 native kidneys in 10 subjects and 10 transplanted kidneys in 10 subjects) performed between January 26, 2010 and July 26, 2010. There were 10 age-matched men and 10 age-matched women (mean $39.8 \pm 12.2$, range $21-60$ years) in this study. Depending on whether the Doppler angle was corrected in the spectral Doppler measurement, Doppler parameters including peak systolic velocity (PSV), end-diastolic velocity (EDV), and resistive index (RI) measured at the interlobar artery of the kidney were divided into two groups, ie, initial Doppler parameters measured without Doppler angle correction (Group 1) and remeasured Doppler parameters with Doppler angle correction (Group 2). Values for PSV, EDV, and RI measured without Doppler angle correction were compared with those measured with Doppler angle correction, and were analyzed statistically with a paired-samples $t$-test.

Results: There were statistical differences in PSV and EDV at the interlobar artery in the upper, mid, and lower poles of the kidney between Group 1 and Group 2 (all $P<0.001$ ). PSV and EDV in Group 1 were significantly lower than in Group 2. RI in Group 1 was the same as that in Group 2 in the upper, mid, and lower poles of the kidneys.

Conclusion: Doppler angle correction plays an important role in the accurate measurement of intrarenal blood flow velocity. The true flow velocity converted from the maximum Doppler velocity shift is produced only when the Doppler angle is $0^{\circ}$, so that the emission sound beam is parallel to the direction of blood flow at the sampled artery. Therefore, the Doppler angle correction should be routinely applied and displayed on renal color duplex sonography.

Keywords: color duplex sonography, blood flow velocity, Doppler angle, Doppler shift, kidney

\section{Introduction}

Intrarenal Doppler parameters, including peak systolic velocity (PSV), end-diastolic velocity (EDV), and resistive index (RI), are considered to be important hemodynamic indicators in screening for renal vascular abnormalities, ${ }^{1,2}$ assessing certain renal conditions, ${ }^{3-5}$ and monitoring the progress and complications of renal transplant allografts. ${ }^{6,7}$ Moreover, in addition to clinical improvement, an indication of the impact on renal hemodynamics resulting from the efficacy of treatment, such as correction of renal artery stenosis or medication for renal transplant rejection, is noninvasively demonstrated by changes in Doppler velocity parameters on renal color duplex sonography. ${ }^{1,7}$ Therefore, an accurate measurement of flow velocity at the intrarenal artery is crucial in performing and interpreting color duplex sonography in native 
and transplanted kidneys. Flow velocity, known as Doppler shift frequency or, simply, the Doppler shift, ${ }^{8}$ is strongly dependent on the insonation angle, ie, the angle between the emission sound beam and flow direction at the sampled artery. It is important to understand that Doppler shift frequency could be $0 \%$ of measured if the Doppler angle is $90^{\circ}$, whereas the flow velocity would be $100 \%$ of measured when the Doppler angle is $0^{\circ}$. In other words, the maximum velocity is produced only when the Doppler angle is $0^{\circ}$ and the sound beam from the transducer is parallel to the direction of blood flow at the sampled artery. ${ }^{9}$

Up until now, Doppler angle correction has not been required in practice guidelines for measuring blood flow velocity at the intrarenal artery during routine color duplex sonography of a transplanted kidney. ${ }^{10}$ In another reference, the Doppler angle correction was needed when measuring acceleration time as an indirect indicator in the diagnosis of renal artery stenosis. ${ }^{11}$

The purpose of our study was to assess the difference in values for intrarenal artery Doppler parameters measured without and with Doppler angle correction. The ultimate goal was to assess whether the Doppler angle correction should be routinely applied for measuring intrarenal Doppler parameters in renal color duplex sonography.

\section{Material and methods}

\section{Patients}

Ten men and ten women matched for age (mean $39.8 \pm 12.2$ [range 21-60] years) were included in this study. There were ten patients (five men and five women) with transplanted kidneys, all located in the right lower quadrant, with a transplant duration ranging from two weeks to five years, who underwent color duplex sonography between January 26, 2009 and July 26, 2010 at the New York Presbyterian Hospital, Weill Cornell Medical Center, New York. Color duplex sonography of 20 native kidneys (ten right kidneys and ten left kidneys in ten healthy subjects, five men and five women) was also performed in Beijing Tongren Hospital, Capital Medical University, Beijing. Clinical indications for renal transplant sonography were fever, hematuria, and pain. Performing color duplex sonography in 20 native kidneys in ten healthy subjects was to be part of routine annual physical examination. All 20 native and ten transplanted kidneys had normal renal function (serum creatinine $<1.1 \mathrm{mg} / \mathrm{dL}$ ). Renal sonography has been requested by nephrologists, transplant surgeons, and emergency or primary care physicians.

Patients with renal failure (serum creatinine $>1.1 \mathrm{mg} / \mathrm{dL}$ ), hydronephrosis, perinephric collection, congestive heart failure, renovascular abnormalities (intrarenal arteriovenous fistula, intrarenal pseudoaneurysm, renal artery stenosis, kidney infarction, renal vein thrombosis), or hypertension (rest brachial artery blood pressure $>140 / 90 \mathrm{mmHg}$ ) were excluded from the study.

\section{Color duplex sonography}

Color duplex sonography was performed with a $2-4 \mathrm{mHz}$ curved linear array or phased array sector transducer (Logiq E9, General Electric, Milwaukee, WI). There was no special preparation prior to renal Doppler sonography. Patients were scanned in the supine or decubitus position. An ultrasound probe covered with transmitting gel was gently placed on the skin over the kidneys. No pressure was applied during scanning to avoid mechanical compression on the kidney and intrarenal vessels. The size of the kidney was measured and its echotexture was observed on high resolution gray scale imaging. Color and spectral Doppler settings, including pulse repetition frequency, total gain, and filter, were adjusted depending on status of blood flow (fast or slow) in the kidneys. The machine settings were considered to be satisfactory when the intrarenal vessel was optimized and the spectrum was clearly displayed. Color flow imaging was used to locate the main renal artery and the interlobar artery at the renal hilum and at the junction of the medulla and pyramids, respectively. A 2-4 mm spectral Doppler gate was routinely placed at the center of the sampled arterial lumen with a lower scale spectral Doppler. We used a Doppler frequency of $3.5 \mathrm{mHz}$ for acquiring the Doppler spectrum and a sweep of $50 \mathrm{~mm} / \mathrm{sec}$ for recording the Doppler spectrum.

The renal color duplex sonography protocol at our institutions includes measurements of PSV (maximal velocity at systole), EDV (minimal velocity at diastole) and RI [(PSV-EDV)/PSV] at the main renal artery, with Doppler angle correction, followed by evaluation of blood flow velocities at the intrarenal arteries. The spectra were sampled at the interlobar arteries in the upper, mid, and lower poles of the kidneys, at which PSV, EDV, and RI were measured with software built into the ultrasound scanner. All images were stored in the picture archiving and communications system in the Department of Radiology, and the review was made on static images only.

The initial measurements of PSV, EDV, and RI at the upper, mid, and lower poles of the native and transplanted kidneys on the spectra were done without displaying the Doppler angle correction (Figure 1). Images with spectral measurements were reviewed with an ultrasound scanner or 


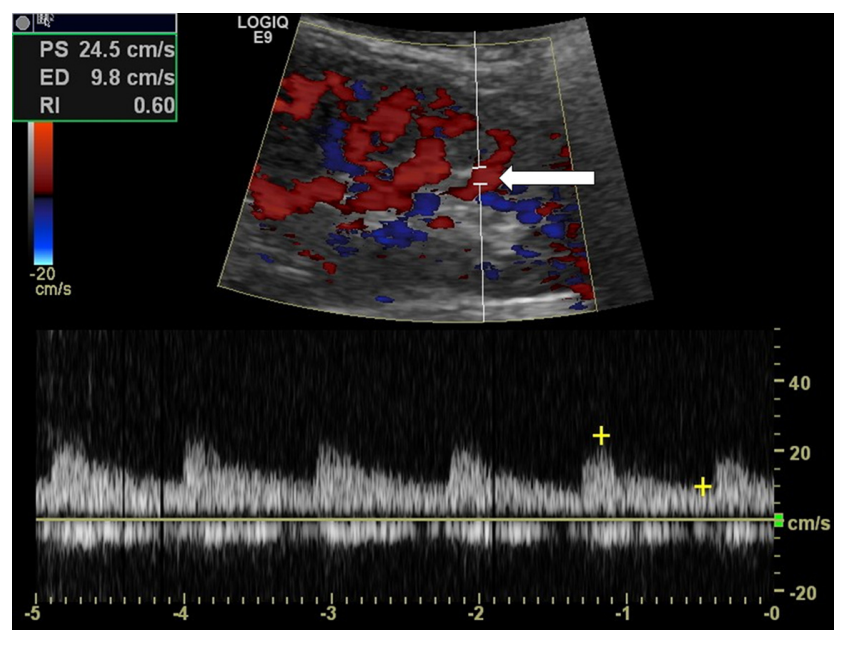

Figure I Longitudinal color duplex sonography of a native kidney with normal function (serum creatinine $0.8 \mathrm{mg} / \mathrm{dL}$ ). Doppler velocities are sampled at the interlobar artery in the mid to lower pole of the kidney with a Doppler gate of $3 \mathrm{~mm}$. Doppler angle correction is not displayed at the Doppler gate (white solid arrow) along the emission sound beam on the image. On this image, the peak systolic velocity, end-diastolic velocity, and resistive index of the interlobar artery are $24.5 \mathrm{~cm} / \mathrm{sec}, 9.8 \mathrm{~cm} / \mathrm{sec}$, and 0.60 , respectively.

the ultrasound reading station of the picture archiving and communications system. When reviewing a static image, Doppler angle correction was displayed and adjusted until the Doppler angle was near $0^{\circ}$, the emission sound beam being parallel to the flow direction of the interlobar artery. The PSV, EDV, and RI were then remeasured and calculated (Figure 2). Finally, all spectral Doppler measurements without and with Doppler angle correction were recorded for statistical analysis.

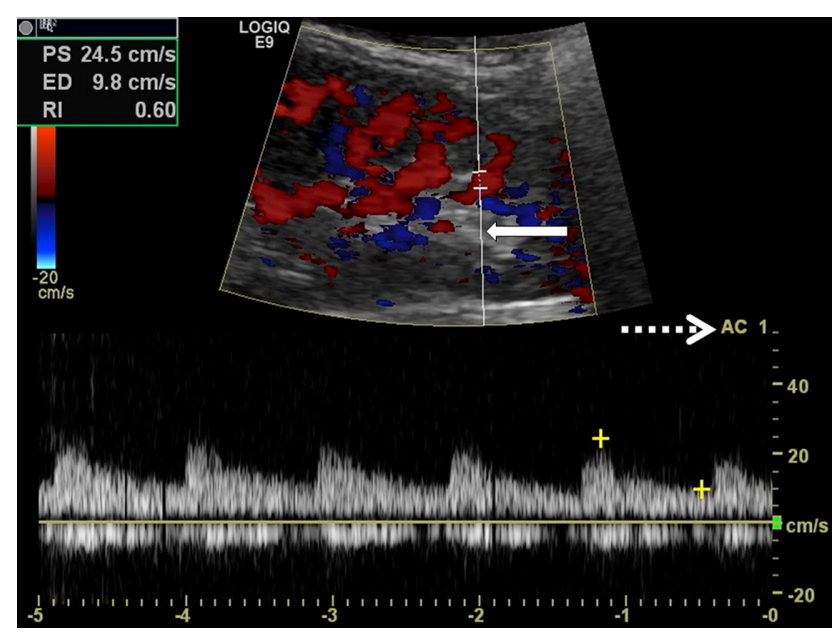

Figure 2A Same static image as in Figure I, although a Doppler angle correction of $1^{\circ}$ appears on the image (white dotted arrow), and the emission sound beam (white arrow) from the transducer is not parallel to the direction of the blood flow. As result, all values of peak systolic velocity, end-diastolic velocity, and resistive index are the same as that measured without Doppler angle correction in Figure I. Therefore, the angle of $\mathrm{I}^{\circ}$ on the image is not the angle between the emission sound beam and the direction of blood flow at the sampled artery, and instead is the angle between the emission sound beam and the Doppler angle correction.

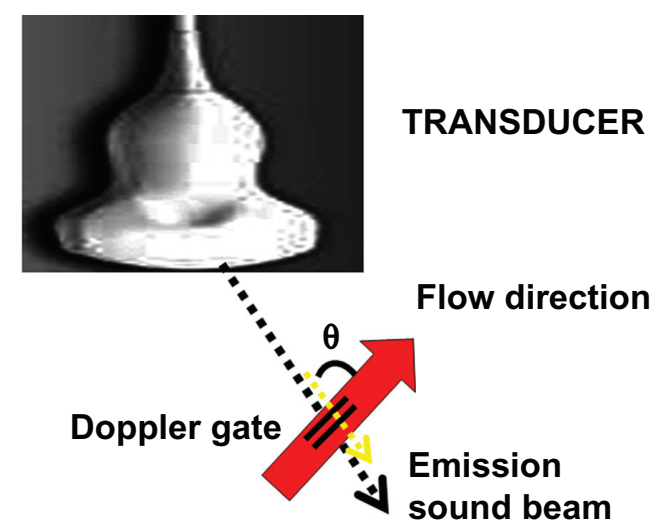

\section{Doppler angle is not corrected}

Figure 2B This illustration shows Doppler angle at the mid to lower pole of the kidney is not corrected. Yellow dotted arrow shows the ultrasound beam emitted from the transducer. The red solid arrow indicates the blood flow direction of the interlobar artery. The white $\Theta$ is the angle between the emission sound beam from the transducer and the flow direction at sampled artery in the mid to lower pole of the kidney.

There was no consent form needed because this was a retrospective study. The institutional review boards at Weill Cornell Medical College and Capital Medical University approved the study. The study complied with the Health Insurance Portability and Accountability Act.

\section{Statistical analysis}

All PSV, EDV, and RI values were expressed as means \pm standard deviations. SPSS software was used to perform the statistical analysis (v 11.5; SPSS Inc, Chicago, IL). A paired-samples $t$-test was used to analyze for statistical differences in values for PSV, EDV, and RI with and without application of Doppler angle correction. Differences in PSV, EDV, and RI values between native and transplanted kidneys were analyzed using the unpaired $t$-test. A $P$ value $<0.05$ was considered a statistically significant difference.

\section{Results}

The largest dimension of the kidneys ranged from $9.5 \mathrm{~cm}$ to $11.8 \mathrm{~cm}(10.21 \pm 1.02 \mathrm{~cm}$ in native kidneys and $10.54 \pm 1.14 \mathrm{~cm}$ in transplanted kidneys). There was no significant difference in Doppler angle-corrected PSV, EDV, or RI at the main renal artery between the native and transplanted kidneys (all $P>0.05$, Table 1). There was no statistical difference in PSV, EDV, and RI values at the interlobar artery measured without or with Doppler angle correction between the native and transplanted kidneys (all $P>0.05$, Table 1 ). However, there was a statistically significant difference in 
Table I Values of Doppler parameters between native and transplanted kidneys

\begin{tabular}{|c|c|c|c|}
\hline $\begin{array}{l}\text { Doppler } \\
\text { parameters }\end{array}$ & $\begin{array}{l}\text { Native kidney }(\mathrm{n}=20) \\
\text { mean } \pm \text { SD }(\mathrm{cm} / \mathrm{sec})\end{array}$ & $\begin{array}{l}\text { Transplanted kidney }(n=10) \text {, } \\
\text { mean } \pm \text { SD }(\mathrm{cm} / \mathrm{sec})\end{array}$ & $P$-value \\
\hline Kidney size $(\mathrm{cm})$ & $10.21 \pm 1.02$ & $10.54 \pm 1.14$ & $>0.05$ \\
\hline PSV, MRA & $79.07 \pm 8.22$ & $80.65 \pm 7.77$ & $>0.05$ \\
\hline EDV, MRA & $29.02 \pm 4.93$ & $29.56 \pm 5.56$ & $>0.05$ \\
\hline PSV, upper pole (I) & $25.54 \pm 5.59$ & $24.09 \pm 6.4 I$ & $>0.05$ \\
\hline (2) & $33.30 \pm 9.21$ & $35.16 \pm 10.21$ & $>0.05$ \\
\hline EDV, upper pole (I) & $10.37 \pm 1.58$ & $9.28 \pm 1.37$ & $>0.05$ \\
\hline$(2)$ & $13.90 \pm 4.14$ & $1 \mathrm{I} .82 \pm 4.14$ & $>0.05$ \\
\hline PSV, mid pole (I) & $27.75 \pm 4.25$ & $28.81 \pm 7.73$ & $>0.05$ \\
\hline$(2)$ & $30.11 \pm 5.18$ & $33.11 \pm 7.55$ & $>0.05$ \\
\hline EDV, mid pole (I) & $10.98 \pm 1.69$ & $9.60 \pm 2.26$ & $>0.05$ \\
\hline (2) & $12.69 \pm 2.17$ & $11.17 \pm 2.05$ & $>0.05$ \\
\hline PSV, lower pole (I) & $23.78 \pm 6.54$ & $22.75 \pm 5.79$ & $>0.05$ \\
\hline (2) & $30.52 \pm 2.81$ & $31.08 \pm 5.35$ & $>0.05$ \\
\hline EDV, lower pole (I) & $9.75 \pm 1.84$ & $8.43 \pm 2.54$ & $>0.05$ \\
\hline (2) & $12.64 \pm 2.85$ & $10.70 \pm 2.09$ & $>0.05$ \\
\hline
\end{tabular}

Notes: Values of Doppler parameters measured in the native and transplanted kidneys. (I) Lists the velocities measured without Doppler angle correction, and (2) lists the velocities measured with Doppler angle correction.

Abbreviations: PSV, peak systolic velocity; EDV, end-diastolic velocity; MRA, main renal artery.

PSV and EDV at the interlobar artery in the upper, mid, and lower poles between the two groups (all $P<0.001$, Table 2). PSV and EDV measured without Doppler angle correction were significantly lower than that measured with Doppler angle correction $(P<0.001)$. RI at the interlobar artery in Group 1 was the same as in Group 2 for the upper, mid, and lower poles of the native and transplanted kidneys (Table 2).

\section{Discussion}

Intrarenal Doppler parameters have been used in the assessment of hemodynamic status in native and transplanted kidneys for decades. ${ }^{1-7}$ As reported, when using the threshold of combining PSV $<15 \mathrm{~cm} / \mathrm{sec}$ at the interlobar artery with the PSV ratio of stenotic renal artery to aorta $>5$, the sensitivity and specificity of color duplex sonography in the diagnosis of renal artery stenosis can reach as high as $91 \%$ and $87 \%$, respectively. ${ }^{2}$ The value of the EDV at the renal parenchyma has an inverse correlation with renal function in the elderly. ${ }^{3}$ Moreover, in our previous report, the PSV and EDV at the intrarenal artery was shown to decrease when significant dysfunction developed in the transplanted kidney. ${ }^{7}$ Therefore, accurate measurements of intrarenal PSV, EDV, and RI are crucial in Doppler sonography of native and transplanted kidneys because a change in the value of Doppler parameters may indicate altered hemodynamic status in the kidney.

We analyzed the PSV, EDV, and RI at the interlobar artery in 30 kidneys measured without and with Doppler angle correction on commercially available ultrasound scanners to assess the accuracy of Doppler velocity measurement by color duplex sonography in native and transplanted kidneys.

Table 2 Intrarenal blood flow velocities measured without and with Doppler angle correction

\begin{tabular}{|c|c|c|c|c|}
\hline $\begin{array}{l}\text { Doppler parameters } \\
\text { at interlobar artery } \\
\text { of kidney }\end{array}$ & $\begin{array}{l}\text { Velocity }(\mathrm{cm} / \mathrm{sec}) \text { measured } \\
\text { without Doppler angle } \\
\text { correction, mean } \pm \text { SD }\end{array}$ & $\begin{array}{l}\text { Velocity }(\mathrm{cm} / \mathrm{sec}) \text { measured } \\
\text { with Doppler angle } \\
\text { correction, mean } \pm \text { SD }\end{array}$ & $t$-test & $P$-value \\
\hline PSV, upper pole & $25.05 \pm 5.25$ & $33.92 \pm 9.76$ & 7.821 & $<0.001$ \\
\hline EDV, upper pole & $9.63 \pm 2.71$ & $12.89 \pm 4.05$ & 7.882 & $<0.001$ \\
\hline RI, upper pole & $0.61 \pm 0.09$ & $0.61 \pm 0.09$ & & \\
\hline PSV, mid pole & $28.10 \pm 5.54$ & $31.11 \pm 6.11$ & 4.296 & $<0.001$ \\
\hline EDV, mid pole & $10.44 \pm 2.83$ & $11.50 \pm 2.78$ & 4.187 & $<0.001$ \\
\hline RI, mid pole & $0.62 \pm 0.10$ & $0.62 \pm 0.10$ & & \\
\hline PSV, lower pole & $23.44 \pm 6.25$ & $30.93 \pm 7.79$ & 7.589 & $<0.001$ \\
\hline EDV, lower pole & $8.86 \pm 2.72$ & $11.63 \pm 3.23$ & 8.763 & $<0.001$ \\
\hline RI, lower pole & $0.62 \pm 0.10$ & $0.62 \pm 0.10$ & & \\
\hline
\end{tabular}

Abbreviations: EDV, end-diastolic velocity; PSV, peak systolic velocity; RI, resistive index; SD, standard deviation. 
According to our results, there was no significant difference in Doppler parameters measured without or with Doppler angle correction between native and transplanted kidneys with normal function (all $P>0.05$ ). The RI measured without Doppler angle correction was the same as that measured with Doppler angle correction. Based on the RI equation, the RI value should be the same if the change in PSV is proportional to the change in EDV, provided that the PSV and EDV are measured with the same Doppler angle.

There was a significant difference at the interlobar artery between PSV and EDV measured without Doppler angle correction (Group 1) and PSV and EDV measured with Doppler angle correction (Group 2). Specifically, PSV and EDV values measured without Doppler angle correction were significantly lower than those measured with Doppler angle correction (all $P<0.001$ ). There are two possible explanations for this statistically significant difference.

The first explanation is related to how blood flow velocity is produced with Doppler sonography. Doppler sonography is used to measure blood flow velocity by the Doppler frequency shift of the echoes from red blood cells. ${ }^{12}$ The standard equation used to convert Doppler frequency shift to velocity is:

$$
\Delta f=\left(2 v f_{0} \cos \Theta\right) / c
$$

where $\Delta f$ is the Doppler shift frequency (the difference between transmitted and received frequencies), $f_{0}$ is the frequency of the incident sound beam from the transducer, $c$ is the speed of sound (assumed to be $1540 \mathrm{~m} / \mathrm{sec}$ in soft tissue), $v$ is the velocity of red blood cells, and $\Theta$ is the angle of the transducer ultrasound beam to flow direction. ${ }^{12,13}$ Hence, three factors that may influence Doppler shift based on the above Doppler equation are: the frequency of the ultrasound beam $\left(f_{0}\right)$; the velocity of red blood cells flowing $(v)$; and the angle of the ultrasound beam to the flow direction $(\Theta)$. The first and second factors are beyond the scope of this report. However, the third factor, $\cos \Theta$, defined as the angle of the ultrasound beam from the transducer to the flow direction, is the focus of this study because the larger the Doppler angle, the smaller the Doppler shift for a given flow speed. ${ }^{8}$

On the basis of the Doppler shift equation, lower scan angles produce larger Doppler shifts. Maximum Doppler frequency shift occurs when blood is flowing directly towards or away from the ultrasound beam. Thus, scanning as close as possible to $0^{\circ}$ will optimize sensitivity and minimize ambiguity in the flow direction. Conversely, as the scan angle approaches $90^{\circ}$, the frequency shift and sensitivity decreases, thereby increasing ambiguity. Hence, blood flowing perpendicular to the ultrasound beam (parallel to the transducer face) will not be displayed, because no Doppler shift is generated. ${ }^{8,13}$ One of the major limitations of any Doppler technique is the dependence of the measurements on the insonation angle between the ultrasound beam and the direction of blood flow. The velocity is underestimated by $6 \%$ at an angle of $20^{\circ}, 13 \%$ at $30^{\circ}, 29 \%$ at $45^{\circ}$, and $0 \%$ at $90^{\circ} .^{14-18}$ This is a common source of technical error and a cause of interobserver variation in measuring blood flow velocity on vascular color duplex sonography. ${ }^{14-16}$

The second explanation lies in how Doppler angle correction works on currently commercially available ultrasound scanners. The Doppler circuitry determines the change in frequency, and this may only be translated into a blood velocity if the Doppler angle is recorded and included in the calculation. Nevertheless, all the newer scanners report blood velocity assuming that the Doppler angle is zero. Given our cases, when Doppler angle correction was not displayed (Figure 1), the Doppler angle would be considered the same as the emission sound beam, ie, the line containing the Doppler gate from the transducer is parallel to the flow direction at the interlobar artery (Figure 2). However, this is more often incorrect than correct, and we are in fact dealing with frequency information unless angle correction has been performed. ${ }^{19}$ Hence, the flow velocity at the interlobar artery of the kidney measured without Doppler angle correction would be inaccurate. This may eventually result in technical error for spectral Doppler sampling if the operator mistakenly assumes the emission sound beam from the transducer is nearly parallel to the blood flow direction without displaying and adjusting the Doppler angle correction. As shown in Table 2, blood flow velocity at the interlobar artery would be underestimated, and blood flow velocity converted from Doppler frequency shift measured without Doppler angle correction (Figure 2A) tends to be lower than that measured with Doppler angle correction (Figure 3A). Without Doppler angle correction, the angle between the flow direction at the interlobar artery to the transducer beam would be $45-60^{\circ}$ in the upper and the lower pole, and $15-30^{\circ}$ in the mid pole (Figure 2B). Because the Doppler angle and flow velocity are directly proportional, the PSV and EDV in Group 1 were significantly lower than that in Group $2(P<0.001)$. This was confirmed by the increased values for PSV and EDV in Group 2 when the Doppler angle was manually corrected from approximately $30^{\circ}$ earlier in the mid to lower pole in Group 1 (Figure 2B) to near $0^{\circ}$ in Group 2 (Figure 3B).

Up until now, we have not found a report in the literature on the importance of Doppler angle correction in the measurement of intrarenal blood flow velocity. 


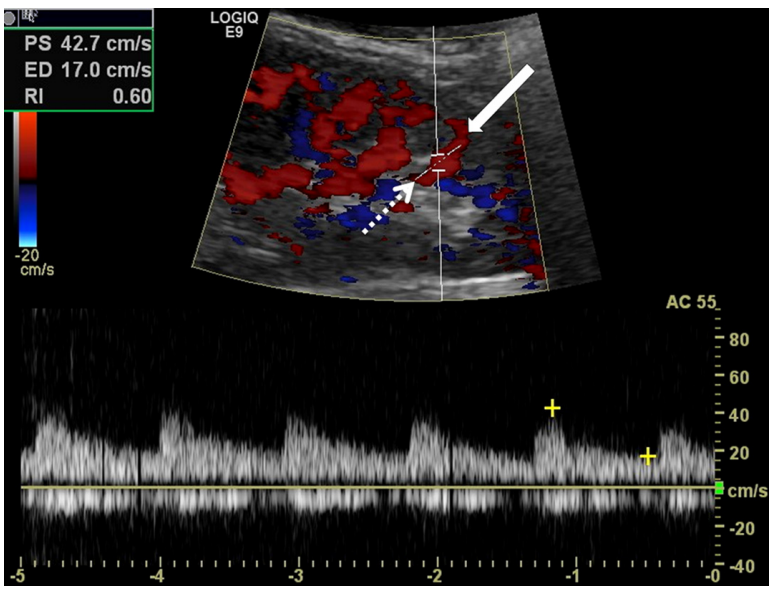

Figure 3A On the same static image as Figure I and Figure 2. The Doppler angle is now corrected to the emission sound beam (white dotted arrow) from the transducer being parallel to the blood flow direction at the sampled interlobar artery (arrow). On remeasurement, the peak systolic velocity and end-diastolic velocity are increased up to $42.7 \mathrm{~cm} / \mathrm{sec}$ and $17.0 \mathrm{~cm} / \mathrm{sec}$, respectively, which are higher than $24.5 \mathrm{~cm} / \mathrm{sec}$ and $9.8 \mathrm{~cm} / \mathrm{sec}$ measured without Doppler angle correction in Figure I. $\mathrm{RI}$ with Doppler angle correction is the same $(0.60)$ as in Figure I. Hence, changing the Doppler angle obviously alters Doppler velocity, whereas it does not affect the resistive index as long as the change of the peak systolic velocity is proportionally to the end diastolic velocity measured at the same Doppler angle.

However, investigations of the relationship between Doppler angle correction and blood flow velocity in assessing the hemodynamics of the cerebral and carotid arteries have been attempted, with the conclusion that Doppler angle correction is crucial in the accurate measurement of velocity in the cerebral ${ }^{14}$ and carotid arteries, ${ }^{15}$ and also in the diagnosis of renal artery stenosis. ${ }^{16}$ Those reports have also

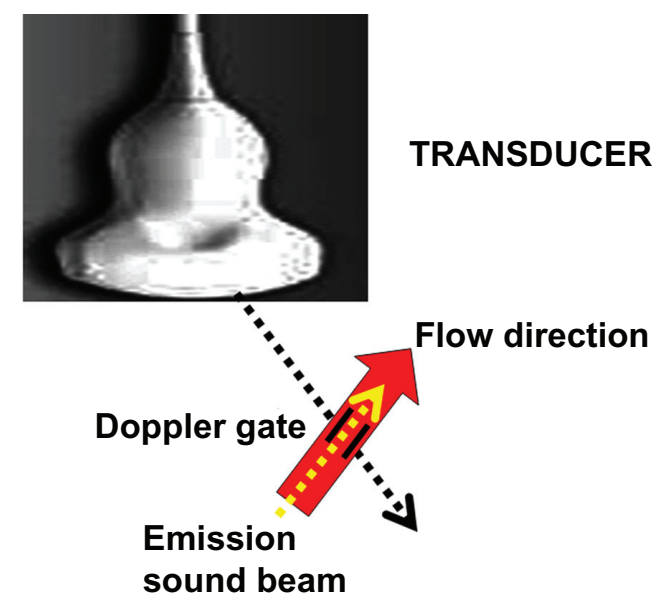

\section{Corrected doppler angle $=0^{\circ}$}

Figure 3B Corrected Doppler angle in the mid to lower pole of the kidney. With Doppler angle correction, the emission sound beam (yellow dotted arrow) from the transducer is parallel to the direction of blood flow (red solid arrow) at the sampled artery. In other words, the Doppler angle between the emission ultrasound beam from the transducer and flow direction at sampled artery has been adjusted from the $\Theta$ in Figure $2 \mathrm{~B}$ to near $0^{\circ}$, which represents a proper Doppler angle correction in renal color duplex sonography. brought attention to the possibility of underestimations in arterial velocity caused by an incorrect Doppler angle, which is considered a technical error made by an individual operator. ${ }^{17,18}$ Underestimating intrarenal artery velocity may result in impaired hemodynamics, which is a source of renal dysfunction, being missed.

The limitations of this retrospective report include intraobserver variation, the different ultrasound scanners that were used, and the small number of cases available for the statistical analysis.

In conclusion, Doppler angle correction is crucial for the accurate measurement of intrarenal blood flow velocity in native and transplanted kidney sonography. The maximum flow velocity is produced only when the Doppler angle between the emitted sound beam and blood flow direction at the sampled artery is $0^{\circ}$. We strongly suggest that Doppler angle correction should be used for all blood flow velocity measurements, not only at the main renal artery, but also at the intrarenal artery. Better knowledge of Doppler principles and familiarity with the Doppler controls on ultrasound scanners would help to improve the quality of renal color duplex sonography. Further research on the importance of Doppler angle correction in the diagnosis of renovascular disease in a larger population is encouraged.

\section{Disclosure}

The authors report no conflicts of interest in this work.

\section{References}

1. Krumme B. Renal Doppler sonography - Update in clinical nephrology. Nephron Clin Pract. 2006;103:24-28.

2. Li JC, Wang L, Jiang YX, et al. Evaluation of renal artery stenosis with velocity parameters of Doppler sonography. $J$ Ultrasound Med. 2006;25:735-742.

3. Pearce JD, Edwards MS, Timothy E, et al. Renal duplex parameters: Blood pressure, and renal function in elderly people. Am J Kidney Dis. 2005; $45: 842-850$

4. Platt JF. Doppler ultrasound of the kidney. Semin Ultrasound CT MR. 1997; 18:22-32.

5. Parolini CP, Noce A, Staffolani E, Giarrizzo GF, Costanzi S, Splendiani G. Renal resistive index and long-term outcome in chronic nephropathies. Radiology. 2009;252:888-896.

6. Baxter GM. Imaging in renal transplantation. Ultrasound Q. 2003;19: 123-138.

7. Gao J, Ng A, Shih G, et al. Intrarenal color Doppler sonography: A window to vascular complications of renal transplants. J Ultrasound Med. 2007;26:1403-1418.

8. Kremkau FW. Doppler principles. Semin Roentgenol. 1992;27:6-16.

9. Hofer M. Teaching Manual of Color Duplex Sonography. 3rd ed. Stuttgart, Germany: Georg Thieme Verlag; 2010.

10. American Institute of Ultrasound in Medicine. Practice guideline for the performance of an ultrasound examination of the abdomen and/ or retroperitoneum. Laurel, MD: American Institute of Ultrasound in Medicine; 2008. Available at: http://www.aium.org/publications/ guidelines/abdominal.pdf. Accessed March 2, 2011. 
11. American College of Radiology. Practice Guideline. Reston, VA: American College of Radiology; 2008.

12. Rubin JM. Spectral Doppler US. Radiographics. 1994;14:139-150.

13. Ralls PW. Power Doppler principles - updated. Available at: http://www nursingcredits.com/sonocredits/article. Accessed September 1, 2010.

14. Zagzebski JA. Essentials of ultrasound physics. Doppler Implement. 1996;5:90-91.

15. Ruma MA. Angle correction can be used to measure peak systolic velocity in the fetal middle cerebral artery. Am J Obstet Gynecol. 2009;200: 397.e1-397.e3.
16. Hadlock J, Beach KW, Strandness DE. Velocity variability in ultrasound Doppler examinations. Ultrasound Med Biol. 2009;35:949-954.

17. Lui EY, Steinman AH, Cobbold RS, Johnston KW. Human factors as a source of error in peak Doppler velocity measurement. J Vasc Surg. 2005;42:972-979.

18. Saad AA, Loupas T, Shapiro LG. Computer vision approach for ultrasound Doppler angle estimation. J Digit Imaging. 2009;22:681-688.

19. Torp-Pedersen ST, Terslev L. Settings and artifacts relevant in color/ power Doppler ultrasound in rheumatology. Ann Rheum Dis. 2008;67: 143-149.

\section{Publish your work in this journal}

The International Journal of Nephrology and Renovascular Disease is an international, peer-reviewed open-access journal focusing on the pathophysiology of the kidney and vascular supply. Epidemiology, screening, diagnosis, and treatment interventions are covered as well as basic science, biochemical and immunological studies. The journal welcomes

\footnotetext{
Submit your manuscript here: http://www.dovepress.com/international-journal-of-nephrology-and-renovascular-disease-journal
}

original research, clinical studies, reviews \& evaluations, expert opinion and commentary, case reports and extended reports. The manuscript management system is completely online and includes a very quick and fair peerreview system, which is all easy to use. Visit http://www.dovepress.com/ testimonials.php to read real quotes from published authors. 